\title{
Lateral Lumbar Interbody Fusion
}

\author{
Abhijit Pawar ${ }^{1}$, Alexander Hughes ${ }^{2}$, Federico Girardi ${ }^{2}$, \\ Andrew Sama ${ }^{2}$, Darren Lebl ${ }^{2}$, Frank Cammisa ${ }^{2}$ \\ ${ }^{I}$ Department of Orthopedic Surgery, Ruby Hall Clinic and Dr. D. Y. Patil Medical College, Pune, India \\ ${ }^{2}$ Department of Orthopedic Surgery, Hospital for Special Surgery, New York, NY, USA
}

The lateral lumbar interbody fusion (LLIF) is a relatively new technique that allows the surgeon to access the intervertebral space from a direct lateral approach either anterior to or through the psoas muscle. This approach provides an alternative to anterior lumbar interbody fusion with instrumentation, posterior lumbar interbody fusion, and transforaminal lumbar interbody fusion for anterior column support. LLIF is minimally invasive, safe, better structural support from the apophyseal ring, potential for coronal plane deformity correction, and indirect decompression, which have has made this technique popular. LLIF is currently being utilized for a variety of pathologies including but not limited to adult de novo lumbar scoliosis, central and foraminal stenosis, spondylolisthesis, and adjacent segment degeneration. Although early clinical outcomes have been good, the potential for significant neurological and vascular vertebral endplate complications exists. Nevertheless, LLIF is a promising technique with the potential to more effectively treat complex adult de novo scoliosis and achieve predictable fusion while avoiding the complications of traditional anterior surgery and posterior interbody techniques.

Keywords: Lateral lumbar fusion; Minimally invasive lumbar spine surgery; Spondylolisthesis

\section{Introduction}

The lateral lumbar interbody fusion (LLIF) technique has shown encouraging results in patients with degenerative conditions of the lumbar spine [1,2]. The advantage of LLIF over traditional anterior approaches is that it avoids exposing the abdominal viscera, large vessels, and sympathetic plexus. Injuries to the nerve roots, dura, and perineural fibrosis, which can occur during posterior lumbar interbody fusion or transforaminal lumbar interbody fusion (TLIF), are minimized with this technique. LLIF can be performed as a stand-alone procedure or as part of circumferential fusion. This technique allows increased surface area for the implant and a superior environment for bone healing [1].

The indications for this technique include adult de novo lumbar scoliosis, central and foraminal stenosis, spondylolisthesis, adjacent segment degeneration, pseudarthrosis, and total disc arthroplasty conversion. This approach can also be used for anterior corpectomy [1,3]. LLIF is useful for revision spine surgeries requiring dissection through scar tissue that is adherent to the dura and neural structures. LLIF shows good results in elderly patients with degenerative scoliosis and significant comorbidities because it indirectly decompresses central and foraminal stenosis and corrects regional and global coronal and sagittal balance. As a result, selective apical fusion in the setting of adult de novo lumbar scoliosis is possible

Received Mar 11, 2015; Revised ; Apr 5, 2015 Accepted Apr 5, 2015

Corresponding author: Abhijit Pawar

Department of Orthopedic Surgery, Ruby Hall Clinic and Dr. D. Y. Patil Medical College,

Sant Tukaram Nagar, Pimpri Pune 411018, India

Tel: +91-73870-80391, Fax: +91-020-278-05217, E-mail: drabhijitpawar@gmail.com 
and superior compared to all posterior constructs. Furthermore, LLIF can be effectively employed in the upper lumbar spine where posterior interbody techniques carry a significant risk due to manipulation of the conus medullaris. Other relative indications are patients at risk for non union due to obesity, osteoporosis, smoking, and previous spine surgeries. This technique is relatively contraindicated if a patient has a low lying L4-L5 disc space, infection, or those with high grade spondylolisthesis due to the location of the lumbar plexus.

\section{Technique}

A left-sided approach is preferred in patients without a coronal plane deformity because of the relatively anterior position of the vasculature on the left side. The spine is preferably approached from the concavity of the scoliotic curve in patients with a coronal plane deformity, as it is possible to approach several levels through a single skin incision (Fig. 1). Furthermore, the table flexion technique helps correct the lumbar deformity. The L4/5 interspace is difficult to access in patients in whom the ipsilateral iliac crest rises above halfway up the L4 vertebrae but can be overcome with table flexion and angled instrumentation.

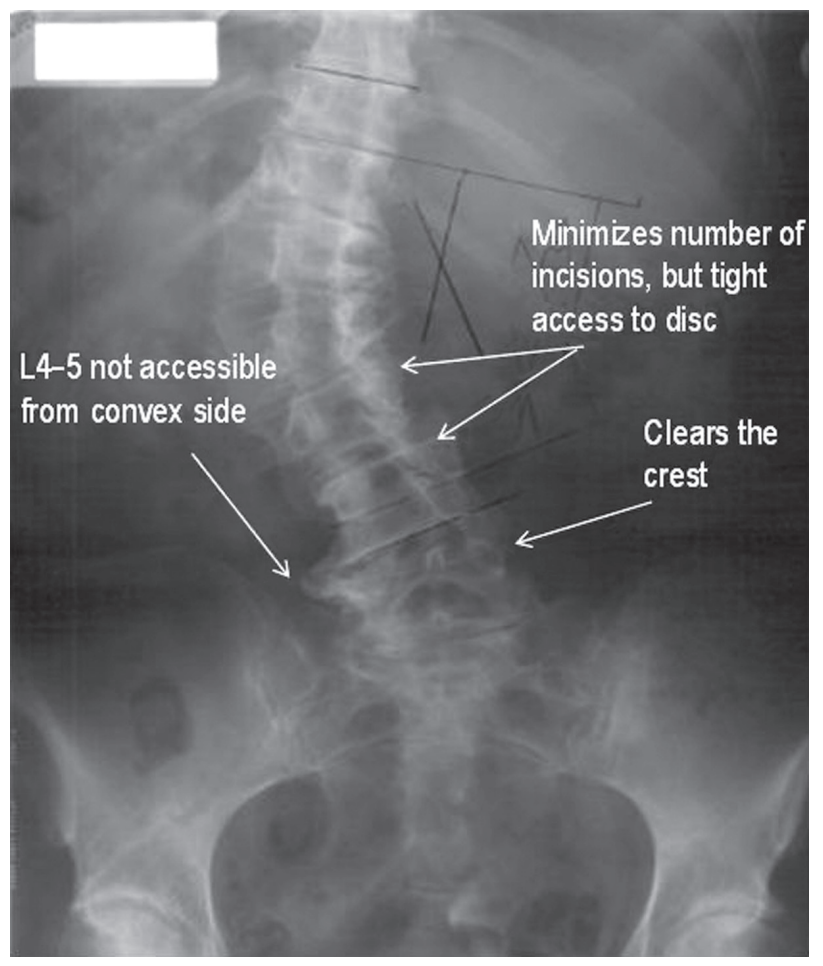

Fig. 1. Planning the lateral lumbar interbody fusion approach.
Preoperative magnetic resonance imaging can be helpful to identify the position of the psoas and the approximate locations of the lumbar plexus and vascular structures, although the operative position (lateral decubitus with hips and knees flexed) is quite different than the supine position $[1,4-7]$.

The patient is placed in the direct lateral decubitus position on the operating table, so the greater trochanter is directly over the table break. The patient is secured to the table with 6 inch wide silk tape on the greater trochanter and the thoracic spine. The surgical table is retroflexed to increase the distance between the iliac crest and the ribs and to gain direct access to the disc space (Fig. 2). True anteroposterior (AP) and lateral fluoroscopic images are obtained. The table can be airplaned so that orthogonal fluoroscopic views are obtained without the need to manipulate the image intensifier. It is imperative to have true AP and lateral images to ensure appropriate endplate preparation and implant insertion. The anterior and posterior borders of the vertebral body and the disc space are marked.

A mini-open technique that permits direct visualization of the retroperitoneal space, psoas muscle, and the neural structures as well as digital palpation of the target disc is utilized at our institution. An oblique incision is made beginning at the anterior, inferior corner of the caudal vertebral body and directed towards the posterior, superior corner of the cephalad vertebral body. The subcutaneous tissue layer is dissected, and the abdominal muscles are split in line with their fibers to minimize injury to the abdominal nerves. The iliohypogastric and the ilioinguinal nerves, which lie between the internal oblique and the transverses abdominis, are protected. The retroperitoneal space is accessed with gentle sweeping of the peritoneum

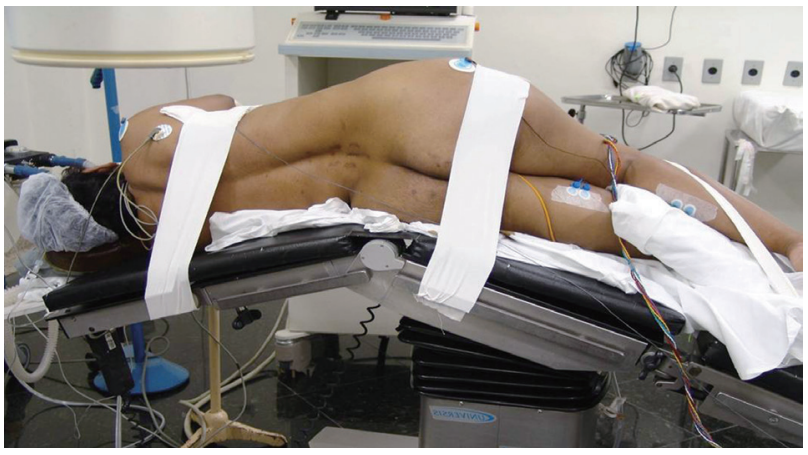

Fig. 2. Lateral positioning with a break in the table at the level of the greater trochanter. 
from posterior to anterior with large sponge sticks to expose the psoas muscle. A transpsoatic dissection is done ventral to the exiting roots. The position of the lumbar plexus is confirmed with neuromonitoring. Retraction is maintained using table-mounted self retaining systems or hand-held renal vein retractors (Fig. 3).

A single Kirschner (K)-wire is introduced half way into the disc space and its position is confirmed on fluoroscopy. We prefer to position the K-wire in the anterior half of the disc space to allow the implant to be prolordotic. A discectomy is performed using pituitary rongeurs, curettes, and paddle shavers. A Cobb elevator is passed along both endplates to the contralateral side using fluoroscopic guidance, which allows for release of the contralateral annulus. The implant is sized by sequential trials and the position is verified on fluoroscopy. Overstuffing of the disc space should be avoided, as it can result in implant subsidence and fracture of the endplate. Numerous graft options exist to fill the cage. The implant is positioned to span the vertebral body and rest on the apophyseal ring. The implant can be left as a stand-alone or a supplemental screw or plate can be fixed depending on the surgeon's preference and the clinical scenario (Fig. 4).

The exposure is irrigated, and the retractors are removed. No drain is required. The transversalis and internal and external obliques are gently closed in layers in an interrupted fashion. The subcuticular layer is sutured with 3-0 monocryl. The anterior fusion can be supplemented by either posterior percutaneous or open pedicle fixation. Fusion is assessed at follow up based on the presence of bridging bone across the disc space and lack of instability across the disc space.

\section{Outcomes and Complications}

Fusion rates and clinical outcomes are predictable after LLIF with minimal complications, and outcomes from this technique compare favorably to other arthrodesis techniques [8]. In a series of 84 patients with a mean 15.7 month follow-up, 68 patients demonstrated solid arthrodesis on a computed tomography scan. Peri- and postoperative complications were $2.4 \%$ and $6.1 \%$, respectively [9]. Another series of 600 patients and 741 levels reported no wound infections, vascular injuries, or intraoperative visceral injuries, but four cases $(0.7 \%)$ of transient postoperative neurologic deficit occurred [3]. Furthermore, they reported minimal complications and no differences between 156 obese and 157 non-obese patients [10]. LLIF indirectly decompresses the neural elements by increasing central canal and foraminal area [11].

LLIF is useful to correct deformities [12]. A significant change in coronal Cobb angle was observed when LLIF
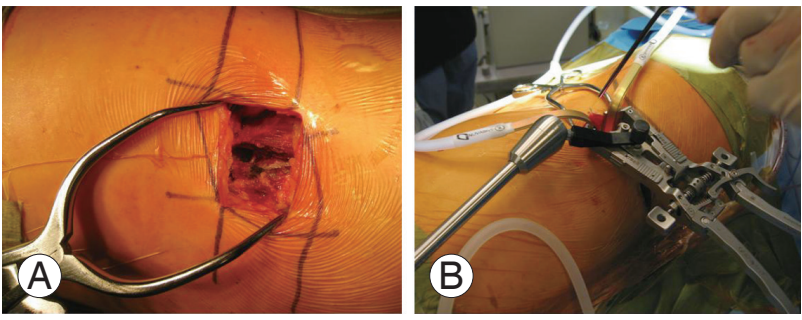

Fig. 3. The mini open approach during lateral lumbar interbody fusion with a table mounted retraction system. (A) Mini open technique of Lateral lumbar interbody fusion. (B) Self retaining tubular retractor used for mini open technique.
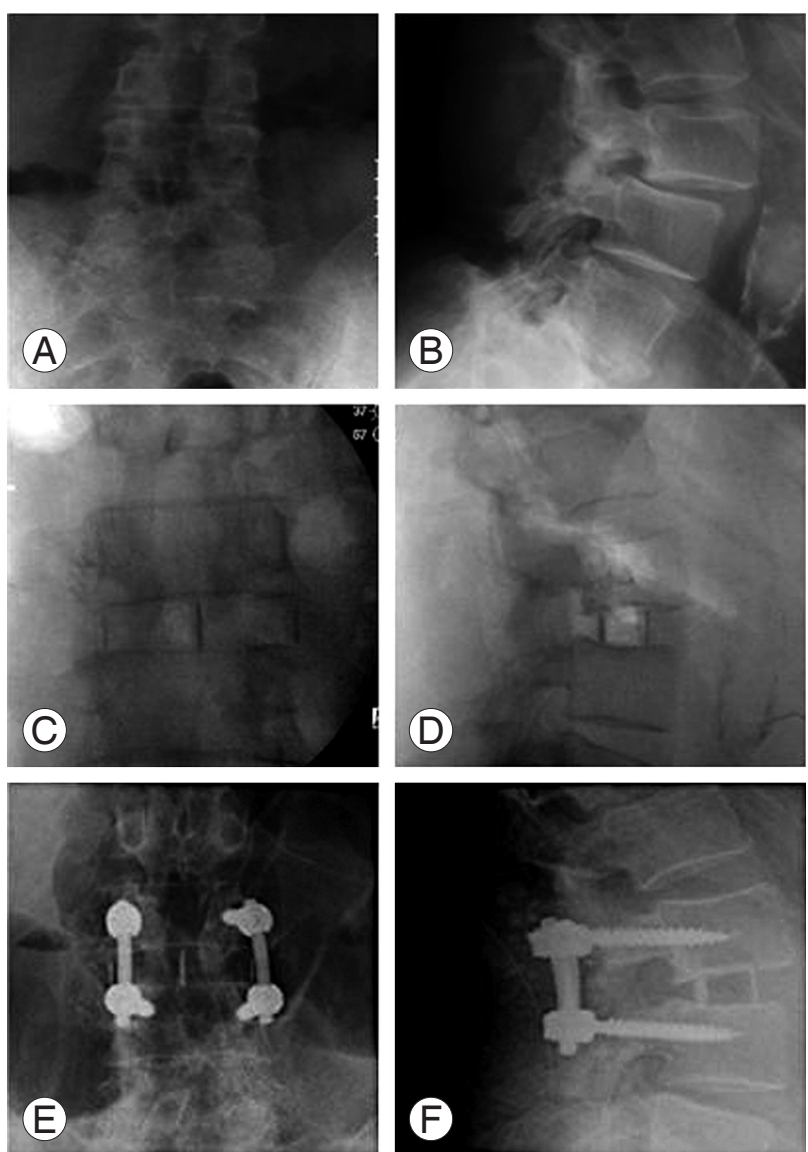

Fig. 4. Case of failed L3-4 decompression and exacerbation of excruciating axial and bilateral $\mathrm{L} 3$ type radicular pain; bilateral $\mathrm{L} 3$ pars fractures treated with lateral lumbar interbody fusion. (A, B) Preoperative radiograph showing Grade I Spondylolisthesis and instability. (C, D) Postoperative radiograph after lateral lumbar interbody fusion. (E, F) Follow radiograph showing fusion. 
was used in a series of 21 patients with a mean follow-up of 21 months. However, sagittal alignment and lumbar lordosis did not improve [13]. Tormenti et al. [14] reported similar results with regard to coronal correction when LLIF was used with posterior fixation. Lumbar lordosis was also preserved in their series. We employ LLIF in a stand-alone fashion and with supplemental fixation (lateral plate, screw, or posterior instrumentation) at Hospital for Special Surgery (New York, NY, USA). We have found excellent reduction in coronal plane deformities. In addition, we have experienced improvements in lumbar lordosis when lordotic implants were used and the implant was placed in the anterior third of the vertebral body. Isaacs et al. [3] reported a major complication rate of $12.1 \%$, which is comparable to rates reported for treating degenerative deformities. Furthermore, complication rates increase in those treated with supplemental open posterior fixation. One of the advantages of LLIF is the ability to insert an implant with a large surface which allows for increased structural support and increased fusion surface area when compared to posterior interbody fusions. LLIF implants are not as large as those used during anterior interbody fusion, their placement avoids exposure and manipulation of the abdominal viscera and vessels. Furthermore, biomechanical studies show that LLIF implants provide a greater reduction in range of motion during flexion, extension, lateral bending, and axial rotation when compared to those of anterior lumbar interbody fusion and TLIF implants $[15,16]$.

The most common complication is anterior thigh pain and numbness, which result from irritating the psoas muscle or transient irritation of the genitofemoral nerve $[1,4,5,8]$. Pumberger et al. [17] examined 181 patients at our institute. No visceral organ injuries or iliac vessel injuries was detected. One patient developed a segmental lumbar artery injury, whereas two had ascending iliolumbar vein injuries. About $38 \%$ of patients had anterior thigh pain 6 weeks postoperatively. This percentage was decreased to $11 \%$ at 12 weeks and to $1 \%$ at 26 weeks. Sensory neurological deficit was seen in $2.2 \%$ of the cases. Mild motor strength was seen in $2.2 \%$ of cases, whereas moderate (3/5) deficit was seen in $2.2 \%$ of cases. No major motor deficits were observed.

Psoas mechanical flexion deficits and lumbar plexusrelated motor deficits have also been reported. We found psoas mechanical flexion deficits in $13.1 \%(\mathrm{n}=32)$ at 6 weeks, $3.7 \%(n=9)$ at 12 weeks, $2.9 \%(n=7)$ at 6 months, and $1.6 \%(\mathrm{n}=4)$ at 12 months. Lumbar plexus-related motor deficits were also seen in $4.9 \%(\mathrm{n}=12)$ at 6 weeks, $4.9 \%(\mathrm{n}=12)$ at 12 weeks, $2.9 \%(\mathrm{n}=7)$ at 6 months, and $2.9 \%(\mathrm{n}=7)$ at 12 months. Female sex and surgery duration were independent risk factors for psoas mechanical flexion deficits, whereas surgery duration was the only independent risk factor for lumbar plexus-related motor deficits.

Other complications that can result after lateral access to the lumbar spine relate to the abdominal wall. Dakwar et al. [18] reported abdominal wall asymmetry leading to bulging of the abdominal wall. This is believed to be a result of denervation of the motor portion of the iliohypogastric and the ilioinguinal nerves supplying the internal oblique and the transverses abdominis muscles. Furthermore the subcostal nerves that originate from the T12 nerve root and innervate the rectus abdominis and the external oblique muscles must be protected.

Complications related to vertebral endplate fractures and subsidence have also been reported $[8,19]$. BrierJones et al. [20] reported four cases of coronal plane and compression fractures of the vertebral body in non-osteoporotic individuals. They concluded that endplate breach, instrumentation, cage movement, and subsidence were contributing factors. We treated two cases of atraumatic vertebral body fracture seen 6 weeks postoperatively [21]. These patients had osteoporosis and received supplemental fixation in the form of screws and/or a lateral plate. As a result, we attempt to avoid placing a screw adjacent to the endplate to prevent a stress riser when using lateral fixation. In addition, we are very cautious when utilizing these implants in a stand-alone fashion in elderly patients.

\section{Conclusions}

LLIF is minimally invasive, indirectly decompresses the neural elements, produces minimal blood loss, and allow for a quicker recovery than other techniques. LLIF is particularly useful in obese individuals where the traditional anterior and the posterior approaches can be technically difficult and associated with high infection rates. Furthermore, the mini-open technique offers a favorable safety profile with rapid localization and disc space preparation. With judicious use of this technique and careful patient selection, a single stage may achieve the goals of surgery while avoiding the increased morbidity associated with a posterior second stage. However, supplemental internal 
fixation or posterior pedicular screw fixation is necessary when further stability is desired. Further prospective case-control analyses are indicated to clearly understand the complications from this technique; however, LLIF is a valuable tool in the armamentarium of the spinal surgeon.

\section{Conflict of Interest}

No potential conflict of interest relevant to this article was reported.

\section{References}

1. Ozgur BM, Aryan HE, Pimenta L, Taylor WR. Extreme lateral interbody fusion (XLIF): a novel surgical technique for anterior lumbar interbody fusion. Spine J 2006;6:435-43.

2. Geisler FH, Blumenthal SL, Guyer RD, et al. Neurological complications of lumbar artificial disc replacement and comparison of clinical results with those related to lumbar arthrodesis in the literature: results of a multicenter, prospective, randomized investigational device exemption study of Charite intervertebral disc. Invited submission from the Joint Section Meeting on Disorders of the Spine and Peripheral Nerves, March 2004. J Neurosurg Spine 2004;1:14354.

3. Isaacs RE, Hyde J, Goodrich JA, Rodgers WB, Phillips FM. A prospective, nonrandomized, multicenter evaluation of extreme lateral interbody fusion for the treatment of adult degenerative scoliosis: perioperative outcomes and complications. Spine (Phila Pa 1976) 2010;35(26 Suppl):S322-30.

4. Guerin P, Obeid I, Bourghli A, et al. The lumbosacral plexus: anatomic considerations for minimally invasive retroperitoneal transpsoas approach. Surg Radiol Anat 2012;34:151-7.

5. Kepler CK, Bogner EA, Herzog RJ, Huang RC. Anatomy of the psoas muscle and lumbar plexus with respect to the surgical approach for lateral transpsoas interbody fusion. Eur Spine J 2011;20:550-6.

6. Guingrich JA, McDermott JC. Ureteral injury during laparoscopy-assisted anterior lumbar fusion. Spine (Phila Pa 1976) 2000;25:1586-8.

7. Rauzzino MJ, Shaffrey CI, Nockels RP, Wiggins GC, Rock J, Wagner J. Anterior lumbar fusion with titanium threaded and mesh interbody cages. Neurosurg
Focus 1999;7:e7.

8. Sharma AK, Kepler CK, Girardi FP, Cammisa FP, Huang RC, Sama AA. Lateral lumbar interbody fusion: clinical and radiographic outcomes at 1 year: a preliminary report. J Spinal Disord Tech 2011;24:24250.

9. Youssef JA, McAfee PC, Patty CA, et al. Minimally invasive surgery: lateral approach interbody fusion: results and review. Spine (Phila Pa 1976) 2010;35(26 Suppl):S302-11.

10. Rodgers WB, Cox CS, Gerber EJ. Early complications of extreme lateral interbody fusion in the obese. J Spinal Disord Tech 2010;23:393-7.

11. Oliveira L, Marchi L, Coutinho E, Pimenta L. A radiographic assessment of the ability of the extreme lateral interbody fusion procedure to indirectly decompress the neural elements. Spine (Phila Pa 1976) 2010;35(26 Suppl):S331-7.

12. Mundis GM, Akbarnia BA, Phillips FM. Adult deformity correction through minimally invasive lateral approach techniques. Spine (Phila Pa 1976) 2010;35(26 Suppl):S312-21.

13. Acosta FL, Liu J, Slimack N, Moller D, Fessler R, Koski T. Changes in coronal and sagittal plane alignment following minimally invasive direct lateral interbody fusion for the treatment of degenerative lumbar disease in adults: a radiographic study. J Neurosurg Spine 2011;15:92-6.

14. Tormenti MJ, Maserati MB, Bonfield CM, Okonkwo DO, Kanter AS. Complications and radiographic correction in adult scoliosis following combined transpsoas extreme lateral interbody fusion and posterior pedicle screw instrumentation. Neurosurg Focus 2010;28:E7.

15. Laws CJ, Coughlin DG, Lotz JC, Serhan HA, Hu SS. Direct lateral approach to lumbar fusion is a biomechanically equivalent alternative to the anterior approach: an in vitro study. Spine (Phila Pa 1976) 2012; 37:819-25.

16. Cappuccino A, Cornwall GB, Turner AW, et al. Biomechanical analysis and review of lateral lumbar fusion constructs. Spine (Phila Pa 1976) 2010;35(26 Suppl):S361-7.

17. Pumberger M, Hughes AP, Huang RR, Sama AA, Cammisa FP, Girardi FP. Neurologic deficit following lateral lumbar interbody fusion. Eur Spine J 2012;21: 1192-9. 
18. Dakwar E, Le TV, Baaj AA, et al. Abdominal wall paresis as a complication of minimally invasive lateral transpsoas interbody fusion. Neurosurg Focus 2011;31:E18.

19. Karikari IO, Grossi PM, Nimjee SM, et al. Minimally invasive lumbar interbody fusion in patients older than 70 years of age: analysis of peri- and postoperative complications. Neurosurgery 2011;68:897-902.
20. Brier-Jones JE, Palmer DK, Inceoglu S, Cheng WK. Vertebral body fractures after transpsoas interbody fusion procedures. Spine J 2011;11:1068-72.

21. Dua K, Kepler CK, Huang RC, Marchenko A. Vertebral body fracture after anterolateral instrumentation and interbody fusion in two osteoporotic patients. Spine J 2010;10:e11-5. 